\title{
Comma in a Coma in Online Headlines: The Case of Daily Post
}

\begin{abstract}
Femi U. Balogun*
*Corresponding Author: Femi U. Balogun,

Abstract: In these days of digital journalism, it would appear that punctuation marks are no longer necessary in writing, especially when casting headlines. But they are! In fact, they are the safety valves that make meanings clear in writing, which when poorly used or not used at all, result in communication breakdown (Balogun, 1995). Consequently, this paper took a synchronic look at some headlines in an online newspaper Daily Post (http://dailypost .us9.list-manage.com), with a view to finding out how well a particular mark - the comma - is used in its headlines. A total number of fifty (50) headlines were purposively sampled from the paper over a period of twelve (12) months, starting from March 2018 and ending in March 2019. They were analyzed using the framework of Error Analysis and it was revealed that the headlines usually conveyed additional unintended meanings which mislead the unwary reader. The flawed headlines were reconstructed and the actual/intended meanings were brought out alongside the unintended meanings which they conveyed. The comma, the most widely used punctuation mark in headlines by this online newspaper, the study concludes, is the most abused by the paper, which sends it to a coma.
\end{abstract}

\section{INTRODUCTION}

Language, the arbitrary vocal symbols by means of which human beings interact, comprises two major skills of production and reception. These two major skills further comprise four sub-skills of writing, speaking, reading and listening. Of these four sub-skills, writing is unarguably the most complex. This is because it is composed of signs and symbols which have to be thoroughly mastered in order to communicate effectively, which is the purpose of language. Although it is not language as such but a tool used to make language readable, writing, like speech, relies within a language system on many of the same structures such as vocabulary, grammar and semantics with the added dependency on a system of signs or symbols (Wikipedia). Bound up with language, it is "a system of more or less permanent marks used to represent an utterance in such a way that it can be recovered more or less exactly without the intervention of the utterer" (Marwa and Sabrina, 2017:17). Writing is, thus, such a difficult task that is restricted to a set of rules and criteria which include organization, coherence, clarity, with accurate language and word choice (Starykey, 2004). This set of rules and criteria serve to make writing meaningful as noncompliance with the rules leads to communication breakdown.

Apart from this set of rules and criteria, there is still another ingredient necessary and pertinent in the business of writing which helps to prevent communication breakdown. It is punctuation. Like every mechanical devise or invention which requires bolts, nuts and safety valves for it to function optimally and prevent a breakdown, collapse or explosion, written communication requires punctuation marks to be effective and to prevent a communication breakdown. Punctuation, explains Wikipedia, is the use of spacing, conventional signs and certain typographical devices as aids to the understanding and correct reading of handwritten and printed text whether read aloud or silently. Thus, it is to written communication what prosodic features or supra-segmental phonemes are to speech or spoken communication. It makes meanings clear when properly applied and when not properly applied, meanings in writing become blurred.

In this digital age when "the internet and its electronic revolutionary spin-offs like e-mail, online speech communities, e-journals, teleconferencing, blogs, tags, the video iPod, iPad, other interface electronic configurations such as widgets, web search and other search engines, provide the necessary platforms and channels for the conduct of e-learning and teaching" (Azuike, 2014:38), it would appear as if punctuation is no longer necessary or relevant. But it is! Without punctuation, written communication would be like a soup without salt. 
This paper, therefore, using a sample corpus collected from Daily Post (dailypost.us9.listmanage.com) for a period of twelve months beginning from March 2018 to March 2019, analyzed some headlines using the framework of error analysis to find out how well a particular punctuation mark - the comma - is being used, especially in casting headlines.

\section{LITERATURE REVIEW}

As noted earlier, punctuation is to writing what prosodic features or supra-segmental phonemes are to speech. The story of punctuation, notes Crystal (2015), goes back over a thousand years - from a time when texts showed no punctuation at all to the present day attention to details. In this digital age, however, the place of punctuation seems to be threatened. This is as revealed by Raphel (2015) who reported David Crystal as saying that in this digital age, there are two extreme views about punctuation as follows: (1) You do not actually need it because it is perfectly possible to write down what you want to say without any punctuation marks or capital letters and people can still read it. (2) Punctuation is essential, not only to avoid ambiguity but also because it shows our identity as educated people. This study subscribes to the second view.

Towards the end of the last century, Balogun (1995) investigated the place of punctuation in students' written communication and came to the conclusion that like prosodic features in speech, punctuation marks will always be relevant in writing. Since the beginning of the $21^{\text {st }}$ Century, this conclusion has not changed even as David Crystal is reported to have pointed out that two extreme views of the subject exist. The common punctuation marks in use in language and especially English grammar still remain: period (.); question mark (?), exclamation point (!); comma (,); semi-colon (;); dash (-); hyphen (-); brackets (( )); braces ([ ]); apostrophe (-'); quotation marks ("')); and ellipsis (...) (Balogun, 1995).

Among researchers who hold the view of Balogun (1995) are Blue (2000), Vietor (2007), Yagoda (2012), Raphel (2015), Broomfield (2016), Oluwadoro (2017).

Yagoda (2012) in particular, who blogs for the Chronicle of Higher Education, has singled out the comma for special attention. He pointed out a lot of errors that could occur as a result of the wrong use or non-use of the comma.

Oluwadoro (2017:4) in his research drew attention to the relevance of punctuation even in the writings of postgraduate students when he noted:

As could be seen in the above, most of the errors arose as result of Students' failure to use a punctuation mark to separate a noun phrasein apposition.

He made this observation after carrying out an error analysis of long essays and theses' acknowledgement by Nigerian students.

By the second decade of the $21^{\text {st }}$ century, a lot of daily newspapers and magazines had gone digital. This has brought in its wake different graphic displays of the contents of the papers and above all different ways of displaying or casting headlines. Whatever the level of sophistication in the display of headlines and bodies of these papers, it does not reduce from the fact that they are all forms of writing, one of the four sub-skills of language and unarguably the most tasking. Consequently, punctuation, an important ingredient in writing will always be relevant in this digital age.

The literature search in this study has confirmed it. The comma, which is the focus of the study, cannot be dispensed with, but must always be used according to rules and specification. This marks out the study as different from any other before it as none has focused on a particular punctuation mark for investigation, using the framework of error analysis.

\section{Method of Data Collection}

Data for this study were purposively sampled from headlines of the Daily Post (http://dailypost. us9.list-manage.com) in the months of March, April, May, December of 2018 and January, February, and March of 2019. The fifty faulty headlines were identified, analyzed and corrected under the following headings as shown in the following table:

Column I: Faulty headlines

Column II: Unintended Meaning/Error 
Column III: Intended Meaning

Column IV: Reconstructed/Correct headline

\section{AIM AND OBJECTIVES OF THE STUDY}

The basic aim of this research was to carry out an error analysis of punctuation errors that are commonly found in headlines of online newspapers with particular reference to Daily Post and focus on the comma.

The objectives are:

- Identify the errors

- Analyze the headlines in which they occur

- Propose what the correct forms should be

- Evaluate the impact of these errors on online headlines, in particular, and digital journalism in general.

- Draw attention of headline casters, editors and reporters to these errors with a view to improving the quality of their headlines.

- Point the attention of teachers to these errors with a view to improving the quality of their teaching (Oluwadoro, 2017).

\section{THEORETICAL FRAMEWORK}

In the field of Applied Linguistics there are the following diagnostic tools: Contrastive Analysis (CA); Error Analysis (EA); and more recently, Needs Analysis (NA). Error analysis (EA) attempted in this study is "the process of determining the incidence, nature, causes and consequences of unsuccessful language" (James, 1998:11). It is a theory replacing the Contrastive Analysis, which was abandoned by linguists and teachers due to its ineffectivity and unreliability. It is one of the key elements of Systemic Grammar which has its roots in the Rationalist Theory of Language learning. Error Analysis involves the systemic interpretation of the unacceptable forms used by someone learning a language (Crystal, 1987). This approach, notes Oluwadono (2017), plays a vital role in the cognitive dimension of language learning.

As Oluwadoro (2017) notes further, the major proponent of this theory is S. Pit Corder, who in his 1967 article titled "The Significance of Learner Errors", gave Error Analysis a new dimension by providing an answer to the question of whether L1 and L2 acquisition processes are the same or not. Ever since, some linguistic scholars have regarded EA as a special variety of Contrastive Analysis (CA). Stockwell (1968), in particular, identified two kinds of CA as a predictive variety and a diagnostic variety, which can be used in the analysis of learners' errors.

This study falls under the diagnostic variety of a CA. Since the 1970s when cognitive approaches to second language learning have been on the increase, EA has attracted a great deal of attention. While some errors are due to the influence of the mother tongue as revealed by contrastive analysis, there are the result of external influence such as inadequate teaching or instructional materials. See Lado (1957) and (1964), Stockwell (1968), Duscova (1969), Gleason (1968), Oluwadoro (2016), among others.

The observation in this study reveals that the punctuation errors identified could be traced to both Sources.

\section{Data Presentation and Analysis}

\begin{tabular}{|c|c|c|c|c|}
\hline 1 & I Faulty Headlines & $\begin{array}{l}\text { II Unintended Meaning/ } \\
\text { Error }\end{array}$ & $\begin{array}{ll}\text { III Intended } \\
\text { Meaning }\end{array}$ & $\begin{array}{l}\text { IV Reconstructed/ } \\
\text { Correct Headlines }\end{array}$ \\
\hline & $\begin{array}{l}\text { *A Veteran actor, Pete } \\
\text { Edochie leads protest } \\
\text { in Port Harcourt } \\
\text { (March 9, 2018) }\end{array}$ & $\begin{array}{l}\text { Two persons - veteran actor } \\
\text { and Pete Edochie meant } \\
\text { here, but the presence of the } \\
\text { singular verb leads tries } \\
\text { prove that it is one person } \\
\text { (Ambiguity) }\end{array}$ & $\begin{array}{l}\text { Only one person } \\
\text { called } \\
\text { who is a veteran actor } \\
\text { leads the protest. }\end{array}$ & $\begin{array}{l}\text { (a) Veteran actor Pete } \\
\text { Edochie leads protest in Port } \\
\text { Harcourt (Note: no comma). } \\
\text { OR } \\
\text { (b) Pete Edochie, veteran actor, } \\
\text { leads protest in Port Harcourt. } \\
\text { (Note: double commas) }\end{array}$ \\
\hline
\end{tabular}




\begin{tabular}{|c|c|c|c|c|}
\hline 2 & $\begin{array}{l}\text { *Former } \text { Governor, } \\
\text { Michika in coma } \\
(\text { March 9, 2018) }\end{array}$ & $\begin{array}{l}\text { Two persons }- \text { a former } \\
\text { governor and another person } \\
\text { called Michika in coma } \\
\text { (Ambiguity) }\end{array}$ & $\begin{array}{l}\text { Only one person } \\
\text { called Michika who } \\
\text { was a former governor } \\
\text { is in coma. }\end{array}$ & $\begin{array}{l}\text { (a) Former Governor Michika } \\
\text { in coma (Note: no comma) } \\
\text { OR } \\
\text { (b) Michika, former governor, } \\
\text { in coma (Note: double } \\
\text { commas) }\end{array}$ \\
\hline 3 & $\begin{array}{l}\text { *PDP Chairman, } \\
\text { Secondus reveals what } \\
\text { must be done to Ibori } \\
\text { (March 11, 2018) }\end{array}$ & $\begin{array}{l}\text { Two persons referred to here, } \\
\text { but the presence of the } \\
\text { singular verb reveals tries to } \\
\text { prove that it is one person } \\
\text { and prevents ambiguity. }\end{array}$ & $\begin{array}{l}\text { Only one person } \\
\text { called Secondus who } \\
\text { is PDP Chairman } \\
\text { reveals what must be } \\
\text { done to Ibori. }\end{array}$ & $\begin{array}{l}\text { (a) PDP Chairman Secondus } \\
\text { reveals what must be done to } \\
\text { Ibori (Note: no comma) } \\
\text { OR } \\
\text { (b) Secondus, PDP } \\
\text { Chairman, reveals what must } \\
\text { be done to Ibori (Note: } \\
\text { double commas). }\end{array}$ \\
\hline 4 & 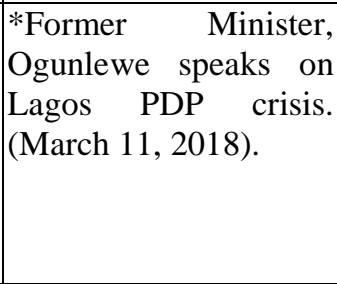 & $\begin{array}{l}\text { Two persons meant - } \\
\text { Ogunlewe and a former } \\
\text { Minister, but the presence of } \\
\text { the singular verb speaks } \\
\text { tends to prove otherwise. }\end{array}$ & $\begin{array}{l}\text { Only one person } \\
\text { called Ogunlewe who } \\
\text { was a former Minister } \\
\text { speaks on Lagos PDP } \\
\text { crisis. }\end{array}$ & $\begin{array}{l}\text { (a) Former Minister Ogunlewe } \\
\text { speaks on Lagos PDP crisis. } \\
\text { (Note: no comma). } \\
\text { OR } \\
\text { (b) Ogunlewe, former Minister, } \\
\text { speaks on Lagos PDP crisis. } \\
\text { (Note: double commas) }\end{array}$ \\
\hline 5 & 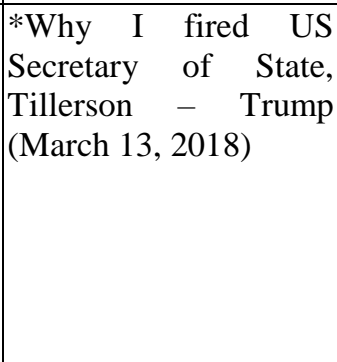 & $\begin{array}{l}\text { Two persons meant here - } \\
\text { Tillerson and US Secretary } \\
\text { of State (Ambiguity) }\end{array}$ & $\begin{array}{l}\text { Only one person } \\
\text { called Tillerson who } \\
\text { is the US Secretary of } \\
\text { State was fired by } \\
\text { Trump. }\end{array}$ & $\begin{array}{l}\text { (a) Why I fired US Secretary } \\
\text { of State Tillerson - Trump. } \\
\text { (Note: no comma) } \\
\text { OR } \\
\text { (b) Why I fired Tillerson, US } \\
\text { Secretary of State. (Note: } \\
\text { one comma separating or } \\
\text { common noun in apposition } \\
\text { to a proper noun). }\end{array}$ \\
\hline 6 & $\begin{array}{l}\text { *Popular actor, Umar } \\
\text { Waragis is dead } \\
(\text { March 13, 2018) }\end{array}$ & $\begin{array}{l}\text { Two persons meant here - } \\
\text { Umar Waragis and a popular } \\
\text { actor, but the presence of the } \\
\text { singular verb is tries to prove } \\
\text { otherwise. (Ambiguity) }\end{array}$ & $\begin{array}{l}\text { Only one person } \\
\text { called Umar Waragis } \\
\text { who is a popular actor } \\
\text { is dead. }\end{array}$ & $\begin{array}{l}\text { (a) Popular actor Umar } \\
\text { Waragis is dead. (Note: no } \\
\text { comma). } \\
\text { OR } \\
\text { (b) Umar Waragis, popular } \\
\text { actor, is dead. (Note: double } \\
\text { commas) }\end{array}$ \\
\hline 7 & $\begin{array}{l}\text { *World's renowned } \\
\text { scientist, } \quad \text { Stephen } \\
\text { Hawking dies at } 76 . \\
(\text { March 13, 2018) }\end{array}$ & $\begin{array}{l}\text { Two persons referred to } \\
\text { here: Stephen Hawking and a } \\
\text { renowned scientist, but the } \\
\text { presence of the singular verb } \\
\text { dies tends to prove } \\
\text { otherwise. } \\
\text { (Ambiguity) }\end{array}$ & 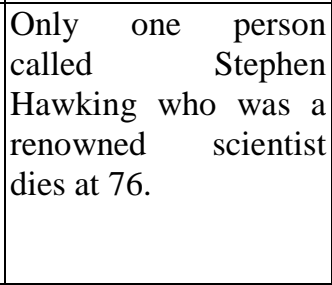 & $\begin{array}{l}\text { (a) Renowned Scientist } \\
\text { Stephen Hawking dies at } 76 . \\
\text { (Note: no comma) } \\
\text { OR } \\
\text { (b) Stephen Hawking, } \\
\text { renowned scientist, dies at } \\
\text { 76. (Note: double commas) }\end{array}$ \\
\hline 8 & $\begin{array}{l}\text { *Buhari reacts to death } \\
\text { of Obasanjo's father- } \\
\text { in-law, Christopher } \\
\text { Abebe (March 23, } \\
\text { 2018) }\end{array}$ & $\begin{array}{l}\text { Two persons referred to } \\
\text { here: Christopher Abebe and } \\
\text { Obasanjo's father-in-law. } \\
\text { (Ambiguity) }\end{array}$ & $\begin{array}{l}\text { Only one person } \\
\text { called Christophr } \\
\text { Abebe who is } \\
\text { Obasanjo's father-in- } \\
\text { law is referred to as } \\
\text { the one who died. }\end{array}$ & $\begin{array}{l}\text { (a) Buhari reacts to death of } \\
\text { Obasanjo's father-in-law } \\
\text { Christopher Abebe. (Note: no } \\
\text { comma). } \\
\text { OR } \\
\text { (b) Buhari reacts to death of } \\
\text { Christopher Abebe, Obasanjo's } \\
\text { father-in-law. (Note: single } \\
\text { comma separating a common } \\
\text { noun in apposition to a proper } \\
\text { noun). }\end{array}$ \\
\hline 9 & $\begin{array}{lc}\text { *Our members } & \text { should } \\
\text { be on looter's } & \text { list - } \\
\text { APC stalwart, } & \text { Frank } \\
\text { blasts Buhari } & \text { govt. } \\
\text { (April 4, 2018). } & \end{array}$ & $\begin{array}{l}\text { Two persons Frank and APC } \\
\text { stalwart meant here, but the } \\
\text { presence of the singular verb } \\
\text { blasts tries to save the } \\
\text { ambiguity. }\end{array}$ & $\begin{array}{l}\text { Only one person } \\
\text { called Frank who is an } \\
\text { APC } \\
\text { Buhari government }\end{array}$ & $\begin{array}{l}\text { (a) Our members should be } \\
\text { on looter's list - APC } \\
\text { stalwart Frank blasts Buhari } \\
\text { (Note: no comma). } \\
\text { OR } \\
\text { (b) Our members should be } \\
\text { on looters' list - Frank, APC }\end{array}$ \\
\hline
\end{tabular}




\begin{tabular}{|c|c|c|c|c|}
\hline & & & & $\begin{array}{l}\text { stalwart, blasts Buhari (Note: } \\
\text { single comma after proper } \\
\text { noun). }\end{array}$ \\
\hline $\mid 10$ & $\begin{array}{l}\text { *Mantu: NAC } \\
\text { chairman, Olapade } \\
\text { Agoro gives INEC 7- } \\
\text { day ultimatum to } \\
\text { deregister PDP, APC, } \\
\text { SDP (April 4, 2018) }\end{array}$ & $\begin{array}{l}\text { Two persons referred to } \\
\text { here: Olapade Agoro and } \\
\text { NAC chairman, but the } \\
\text { presence of the singular verb } \\
\text { gives in the headline tries to } \\
\text { prevent the ambiguity error. }\end{array}$ & $\begin{array}{l}\text { Only one person } \\
\text { called Olapede Agoro } \\
\text { who is the NAC } \\
\text { chairman gives the } 7- \\
\text { day ultimatum. }\end{array}$ & $\begin{array}{l}\text { (a Mantu: NAC chairman } \\
\text { Olapade Agoro gives INEC } \\
\text { 7-day ultimatum to } \\
\text { deregister PDP, APC, SDP. } \\
\text { (Note: no comma) } \\
\text { OR } \\
\text { (b) Mantu: Olapade Agoro, } \\
\text { NAC chairman, gives INEC } \\
\text { 7-day ultimatum to } \\
\text { deregister PDP, APC, SDP } \\
\text { (Note: double commas). }\end{array}$ \\
\hline \begin{tabular}{|l|}
11 \\
\end{tabular} & $\begin{array}{lr}\text { *MASSOB } & \text { warns Igbo } \\
\text { ahead of election, } \\
\text { reveals } & \text { what } \\
\text { herdsmen, } & \text { Boko } \\
\text { Haram will do } & \text { (April } \\
4,2018) & \end{array}$ & $\begin{array}{l}\text { Two commas wrongly used } \\
\text { instead of one. A comma } \\
\text { cannot separate independent } \\
\text { clauses. It is a semi-colon } \\
\text { that can. (comma splice } \\
\text { error). }\end{array}$ & - & $\begin{array}{l}\text { MASSBO warns Igbo ahead } \\
\text { of election; reveals what } \\
\text { herdsmen, Boko Haram will } \\
\text { do. (Note: semi-colon is used } \\
\text { to separate two clauses) }\end{array}$ \\
\hline 12 & $\begin{array}{l}\text { *Gunmen kidnap VOA } \\
\text { reporter's wife, child } \\
\text { in Kaduna, murder } \\
\text { FRSC official (March } \\
1,2018 \text { ). }\end{array}$ & $\begin{array}{l}\text { Two commas wrongly used } \\
\text { instead of one. A comma } \\
\text { cannot be used to separate } \\
\text { independent clauses. It is a } \\
\text { semi-colon that can be used. } \\
\text { (comma splice error) }\end{array}$ & - & $\begin{array}{l}\text { Gunmen kidnap VOA } \\
\text { reporter's wife, child; murder } \\
\text { FRSC official. } \\
\text { (Note: semi-colon is used to } \\
\text { separate two clauses) }\end{array}$ \\
\hline 13 & $\begin{array}{l}\text { *I won past elections } \\
\text { without big politicians, } \\
\text { only God raised me - } \\
\text { Okorocha } \\
\text { (April } 4,2018)\end{array}$ & $\begin{array}{l}\text { A comma wrongly used to } \\
\text { separate independent clauses. } \\
\text { (comma splice error). }\end{array}$ & - & $\begin{array}{l}\text { I won past elections without } \\
\text { big politicians; only God } \\
\text { raised me - Okorocha. } \\
\text { (Note: semi-colon is used to } \\
\text { separate two clauses) }\end{array}$ \\
\hline 14 & $\begin{array}{l}\text { *Ganduje ready to } \\
\text { work with Kwankwaso } \\
\text { - Kano APC chairman, } \\
\text { Abbas (May 30, 2018) }\end{array}$ & $\begin{array}{l}\text { Two persons referred to } \\
\text { here: Abbas and APC } \\
\text { chairman (Ambiguity) }\end{array}$ & $\begin{array}{l}\text { Only one person - } \\
\text { Abbas who is the APC } \\
\text { chairman made the } \\
\text { statement that } \\
\text { Ganduje is ready to } \\
\text { work with } \\
\text { Kwankwaso. }\end{array}$ & $\begin{array}{l}\text { (a) Ganduje read to work } \\
\text { with Kwankwaso - APC } \\
\text { chairman Abbas (Note: no } \\
\text { comma). } \\
\text { OR } \\
\text { (b) Ganduje ready to work } \\
\text { with Kwankwaso - Abbas, } \\
\text { APC chairman. (Note: } \\
\text { comma after proper noun in } \\
\text { apposition). }\end{array}$ \\
\hline 15 & $\begin{array}{l}\text { *ZLP guber candidate, } \\
\text { Alli slams Gov } \\
\text { Ajimobi over security } \\
\text { levies on churches, } \\
\text { mosques in Oyo State. } \\
(\text { Dec. 29, 2018) }\end{array}$ & $\begin{array}{l}\text { Two persons meant here: } \\
\text { Alli and ZLP guber } \\
\text { candidate, but the presence } \\
\text { of the singular verb slams } \\
\text { tends to save the headline } \\
\text { from ambiguity. }\end{array}$ & $\begin{array}{l}\text { Only one person } \\
\text { Alli who is also the } \\
\text { ZLP guber candidate } \\
\text { slams Gov. Ajimobi } \\
\text { over security levies on } \\
\text { churches and mosques } \\
\text { in Oyo State. }\end{array}$ & $\begin{array}{l}\text { (a) ZLP guber candidate Alli } \\
\text { slams Gov. Ajimobi over } \\
\text { security levies on churches, } \\
\text { mosques in Oyo State (Note: } \\
\text { no comma). } \\
\text { OR } \\
\text { (b) Alli, ZLP guber } \\
\text { candidate, slams Gov. } \\
\text { Ajimobi over security levies } \\
\text { on churches, mosques. } \\
\text { (Note: double commas) }\end{array}$ \\
\hline 16 & $\begin{array}{l}\text { *Why Yoruba will } \\
\text { vote for Buhari, APC - } \\
\text { Campaign director Oke } \\
\text { (January 3, 2019) }\end{array}$ & $\begin{array}{l}\text { Two persons referred to } \\
\text { here: Oke and campaign } \\
\text { director give reasons why } \\
\text { Yoruba will vote for Buhari } \\
\text { and APC. Ambiguity is } \\
\text { here! }\end{array}$ & $\begin{array}{l}\text { Only one person - } \\
\text { Oke who is the } \\
\text { campaign director } \\
\text { gives reasons why } \\
\text { Yoruba will vote for } \\
\text { Buhari and APC. }\end{array}$ & $\begin{array}{l}\text { (a) Why Yoruba will vote for } \\
\text { Buhari, APC - Campaign } \\
\text { director Oke. (Note: no } \\
\text { comma) } \\
\text { OR } \\
\text { (b) Why Yoruba will vote for } \\
\text { Buhari, APC - Oke, } \\
\text { Campaign director. (Note: a } \\
\text { comma after proper noun } \\
\text { and before common noun). }\end{array}$ \\
\hline
\end{tabular}




\begin{tabular}{|c|c|c|c|c|}
\hline 17 &  & $\begin{array}{l}\text { Two persons referred to } \\
\text { here: Akinlade and } \\
\text { Amosun's candidate; the } \\
\text { presence of the singular verb } \\
\text { speaks, however tries to } \\
\text { prevent the ambiguity. }\end{array}$ & $\begin{array}{l}\text { Only one person }- \\
\text { Akinlade who is } \\
\text { Amosun's candidate, } \\
\text { speaks ahead of guber } \\
\text { battle. }\end{array}$ & $\begin{array}{l}\text { (a) APC vs APM: Amosun's } \\
\text { candidate Akinlade speaks } \\
\text { ahead of Ogun guber battle. } \\
\text { (Note: no comma). } \\
\text { OR } \\
\text { (b) APC vs APM: Akinlade, } \\
\text { Amosun's candidate, speaks } \\
\text { of Ogun guber battle. (Note: } \\
\text { double commas) }\end{array}$ \\
\hline 18 & \begin{tabular}{lrr}
$* 2019$ & Presidency: \\
What & Femi-Kayode \\
said as & INEC & names \\
Buhari’s & niece, & Amina \\
Zakari & head & of \\
Collation & \multicolumn{2}{c}{ Centre } \\
(January 3,2019$)$
\end{tabular} & $\begin{array}{l}\text { Two persons referred to } \\
\text { here: Amina Zakari and } \\
\text { Buhari's niece (Ambiguity). }\end{array}$ & $\begin{array}{l}\text { Only one person - } \\
\text { Amina Zakari, who is } \\
\text { Buhari's niece, is the } \\
\text { one named head of } \\
\text { INEC } \\
\text { Centre }\end{array}$ & $\begin{array}{l}\text { (a) } 2019 \text { Presidency: Wh } \\
\text { Femi-Kayode said as INE } \\
\text { names Buhari's niece Amin } \\
\text { Zakari as head of Collatio } \\
\text { Centre (Note: no comma). } \\
\text { OR } \\
\text { (b) } 2019 \text { Presidency: Wh } \\
\text { Femi-Kayode said as INE } \\
\text { names Amina Zakari, Buhari } \\
\text { niece, head of collation cent } \\
\text { (Note: double commas) }\end{array}$ \\
\hline 19 & $\begin{array}{l}\text { *Atiku's promise of } \\
\text { restructuring a sham - } \\
\text { Ex PDP Chairman, } \\
\text { Salvador (Jan 4,2019) }\end{array}$ & $\begin{array}{l}\text { Two persons referred to } \\
\text { here: Salvador and Ex PDP } \\
\text { chairman. (Ambiguity) }\end{array}$ & $\begin{array}{l}\text { Only one person } \\
\text { called Salvador, who } \\
\text { is Ex-PDP chairman is } \\
\text { meant. }\end{array}$ & $\begin{array}{l}\text { (a) Atiku's promise } \\
\text { restructuring a sham - E } \\
\text { PDP chairman Salvado } \\
\text { (Note: no comma). } \\
\text { OR } \\
\text { (b) Atiku's promise c } \\
\text { restructuring a sham } \\
\text { Salvador, Ex PDP chairmar } \\
\text { (Note: one comma). }\end{array}$ \\
\hline 20 & $\begin{array}{l}\text { *New York Prophecy: } \\
\text { Adegboye r snubs } \\
\text { Atiku, reveals who'll } \\
\text { take over from Buhari, } \\
\text { speaks on crisis, Boko } \\
\text { Haram. (January 4, } \\
2019)\end{array}$ & $\begin{array}{l}\text { Comma wrongly used to } \\
\text { separate independent clauses, } \\
\text { common nouns and proper } \\
\text { nouns. (Comma splice) }\end{array}$ & - & $\begin{array}{l}\text { New York } \\
\text { Adegboye Prophecy } \\
\text { reveals who'll take over fron } \\
\text { Buhari; speaks on crisis } \\
\text { Boko Haram. (Use semi } \\
\text { colon } \\
\text { independent clauses). }\end{array}$ \\
\hline 21 & 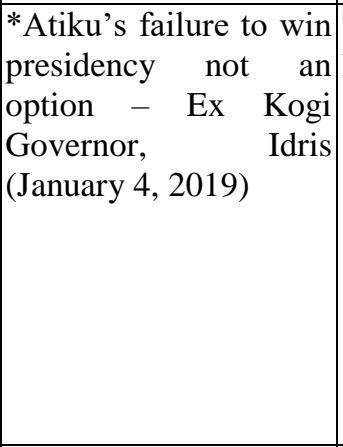 & $\begin{array}{l}\text { Two persons referred to } \\
\text { here: Idris and Ex-Kogi } \\
\text { Governor. (Ambiguity) }\end{array}$ & $\begin{array}{llr}\text { Only one person } \\
\text { called Idris who is Ex- } \\
\text { Kogi } & \text { Governor is } \\
\text { meant. } & \end{array}$ & $\begin{array}{l}\text { (a) Atiku's failure to } \\
\text { presidency not an option - Ex } \\
\text { Kogi Governor Idris. (Note: } \\
\text { comma, common noun occu } \\
\text { before proper noun). } \\
\text { OR } \\
\text { (b) Atiku's failure to w } \\
\text { presidency not an option } \\
\text { Idris, Ex-Kogi Governo } \\
\text { (Note: a comma after prope } \\
\text { noun Idris). }\end{array}$ \\
\hline 22 & $\begin{array}{l}\text { *NGO petitions Oyo } \\
\text { CP, fingers PPRO, } \\
\text { DPO over alleged poor } \\
\text { handling of rape case } \\
\text { (January } 6,2019)\end{array}$ & $\begin{array}{l}\text { comma wrongly used to } \\
\text { separate independent clause } \\
\text { and common noun. (Comma } \\
\text { splice) }\end{array}$ & - & $\begin{array}{l}\text { NGO petitions Oyo CP; finge } \\
\text { PPRO, DPO over allege } \\
\text { handling of rape case. ( } U \text {, } \\
\text { semi-colon to separa } \\
\text { independent clause). }\end{array}$ \\
\hline 23 & \begin{tabular}{|lr}
$*$ President & Buhar \\
reacts to & death of \\
former & Kano \\
Governor, & Abdullahi, \\
sends & delegation. \\
(January 6, 2019)
\end{tabular} & $\begin{array}{l}\text { Comma wrongly used to } \\
\text { separate independent clause } \\
\text { from proper noun (comma } \\
\text { splice). }\end{array}$ & - & $\begin{array}{l}\text { President Buhari reacts } \\
\text { death of former Kan } \\
\text { Governor Abdullahi; senc } \\
\text { delegation. (Semi-colon use } \\
\text { to separate independe } \\
\text { clause). }\end{array}$ \\
\hline 24 & 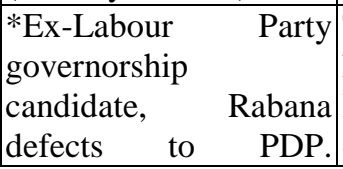 & $\begin{array}{l}\text { Two persons referred to } \\
\text { here: Rabana and Ex-Labour } \\
\text { Party governorship } \\
\text { candidate. (Ambiguity). }\end{array}$ & $\begin{array}{lr}\text { Only one } & \text { person } \\
\text { called Rabana } & \text { who is } \\
\text { a Ex-Labour } & \text { Party } \\
\text { governorship } & \end{array}$ & $\begin{array}{ll}\text { (a) Ex-Labour } & \text { Par } \\
\text { governorship } & \text { candida } \\
\text { Rabana defects } & \text { to PD } \\
(\text { Note: } \text { no comma }) & \end{array}$ \\
\hline
\end{tabular}




\begin{tabular}{|c|c|c|c|c|}
\hline & (January 6, 2019). & & candidate is meant. & $\begin{array}{l}\text { OR } \\
\text { (b) Rabana, Ex-Labour Party } \\
\text { governorship candidate, } \\
\text { defects to PDP. (Note: } \\
\text { double commas). }\end{array}$ \\
\hline 25 & $\begin{array}{l}\text { *2019: Amaechi attests } \\
\text { to insecurity under } \\
\text { Buhari, says he can't } \\
\text { live in Gwarimpa, } \\
\text { Utako. (January 11, } \\
\text { 2019). }\end{array}$ & $\begin{array}{l}\text { Comma wrongly used to } \\
\text { separate independent clauses } \\
\text { (common splice) }\end{array}$ & & $\begin{array}{l}\text { Amaechi attests to insecurity; } \\
\text { says he can't live in Gwarimpa, } \\
\text { Utako. (Note: a semi-colon } \\
\text { instead of a comma is used to } \\
\text { separate the two independent } \\
\text { clauses in the headline). }\end{array}$ \\
\hline 26 & $\begin{array}{l}\text { *N10,000 can't do } \\
\text { anything for traders - } \\
\text { SDP guber candidate, } \\
\text { Ayorinde slams Buhari } \\
\text { over traders money } \\
\text { scheme (January 11, } \\
2019)\end{array}$ & $\begin{array}{l}\text { Two persons referred to } \\
\text { here: Ayorinde and SDP } \\
\text { guber } \\
\text { (Ambiguity) }\end{array}$ & $\begin{array}{l}\text { Only one person } \\
\text { called Ayorinde who } \\
\text { is the SDP guber } \\
\text { candidate is meant. }\end{array}$ & $\begin{array}{l}\text { N10,000 can't do anything } \\
\text { for traders - SDP guber } \\
\text { candidate Ayorinde slams } \\
\text { Buhari over trader money } \\
\text { scheme. (Note: no comma) }\end{array}$ \\
\hline 27 & $\begin{array}{l}\text { *Enugu } \quad \text { Guber: } \\
\text { People's support for } \\
\text { Ugwanyi } \\
\text { overwhelming }- \text { PFN } \\
\text { Chairman, Madu. } \\
\text { (January 11, 2019). }\end{array}$ & $\begin{array}{l}\text { Two persons referred to } \\
\text { here: Madu and PFN } \\
\text { chairman (Ambiguity) }\end{array}$ & $\begin{array}{lll}\text { Only } & \text { one } & \text { person } \\
\text { called } & \text { Madu who is } \\
\text { RFN } & \text { chairman is } \\
\text { meant. } & & \end{array}$ & 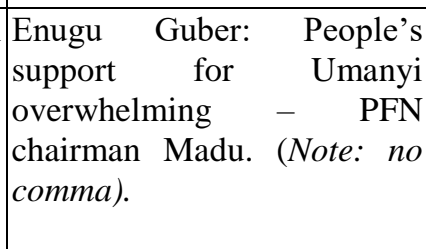 \\
\hline 28 & $\begin{array}{l}\text { *2019 Presidency: } \\
\text { Keyamo speaks as } \\
\text { Atiku campaign DG, } \\
\text { Umar Kumo dumps } \\
\text { PDP for APC. (January } \\
\text { 12, 2019). }\end{array}$ & $\begin{array}{l}\text { Two persons referred to } \\
\text { here: Umar Kumo and } \\
\text { Atiku's campaign DG. } \\
\text { (Ambiguity) }\end{array}$ & $\begin{array}{llr}\text { Only one } & \text { person } \\
\text { called Umar } & \text { Kumo } \\
\text { who is } & \text { Atiku's } \\
\text { campaign } & \text { DG is } \\
\text { meant. } & & \end{array}$ & $\begin{array}{l}\text { (a) } 2019 \text { Presidency: Keyamo } \\
\text { speaks as Atiku's campaign } \\
\text { DG Umar Kumo dumps PDP } \\
\text { to APC. (Note: no comma) } \\
\text { OR } \\
\text { (b) } 2019 \text { Presidency: Keyamo } \\
\text { speaks as Umar Kumo, Atiku's } \\
\text { campaign DG, dumps PDP for } \\
\text { APC. (Note: double commas). }\end{array}$ \\
\hline 29 & $\begin{array}{l}\text { *Jonathan's ex-aid, } \\
\text { Omokri reacts to } \\
\text { Buhari government's } \\
\text { plan to arraign CJN } \\
\text { Onoghen. (January 12, } \\
\text { 2019) }\end{array}$ & $\begin{array}{l}\text { Two persons referred to } \\
\text { here: Omokri and Jonathan's } \\
\text { ex-aid. (Ambiguity) }\end{array}$ & $\begin{array}{l}\text { Only one person } \\
\text { called Omokri who is } \\
\text { Jonathan's ex-aid is } \\
\text { meant. }\end{array}$ & $\begin{array}{l}\text { (a) Jonathan's ex-aid Omokri } \\
\text { reacts to Buhari government's } \\
\text { plan to arraign CJN Omoghen } \\
\text { (Note: no comma). } \\
\quad \text { OR } \\
\text { Omokri, Jonathan's ex-aid } \\
\text { reacts to Buhari government's } \\
\text { plan to arraign CJN Omoghen. } \\
\text { (Note: double commas). }\end{array}$ \\
\hline 30 & $\begin{array}{lr}* \text { Abia } & 2019: \\
\text { Oshiomholer attacks } \\
\text { APC chieftain, Ikechi } \\
\text { Emenike over romance } \\
\text { with Gov. Ikpeazu. } \\
\text { (January } 15,2019 .\end{array}$ & $\begin{array}{l}\text { Two persons referred to } \\
\text { here: Ikechi Emenike and } \\
\text { APC chieftain. (Ambiguity) }\end{array}$ & $\begin{array}{l}\text { Only one person } \\
\text { called Ikechi Emenike } \\
\text { who is an APC } \\
\text { chieftain is meant. }\end{array}$ & $\begin{array}{l}\text { (a) Oshiomhole attacks APC } \\
\text { chieftain Ikechi Emenike over } \\
\text { romance with Gov Ikpeazu. } \\
\text { (Note: no comma). } \\
\text { OR } \\
\text { (b) Oshiomhole attacks Ikechi } \\
\text { Emenike, APC chieftain, over } \\
\text { romance with Gov. Ikpeazu. } \\
\text { (Note: double commas). }\end{array}$ \\
\hline 31 & $\begin{array}{l}\text { *Police wanted to kill } \\
\text { me, everything I said } \\
\text { was under duress } \\
\text { (January 16, 2019) }\end{array}$ & $\begin{array}{l}\text { Comma wrongly used to } \\
\text { separate two independent } \\
\text { clauses (Comma splice). }\end{array}$ & & $\begin{array}{l}\text { Police wanted to kill me; } \\
\text { everything I said was under } \\
\text { duress. (Note: a semi-colon } \\
\text { instead of a comma). }\end{array}$ \\
\hline 32 & $\begin{array}{l}\text { *DG Taskforce on } \\
\text { Prohibition of } \\
\text { importation of Arms, } \\
\text { Okereke speaks on } \\
\text { new Police IG, Adams. } \\
\text { (January 27, 2019). }\end{array}$ & $\begin{array}{l}\text { Four persons referred to here } \\
\text { instead of two: (i) Okereke } \\
\text { (ii) DG Taskforce (iii) } \\
\text { Adamu (iv) Police } \\
\text { (Ambiguity). }\end{array}$ & $\begin{array}{l}\text { Two persons: Okereke } \\
\text { who is the DG } \\
\text { Taskforce } \\
\text { Prohibition on } \\
\text { Adamu who is the } \\
\text { new Police IG are } \\
\text { meant. }\end{array}$ & $\begin{array}{l}\text { (a) DG Taskforce on } \\
\text { Prohibition of Importation of } \\
\text { Arms Okereke speaks on } \\
\text { new Police IG Adamu. } \\
\text { (Note: no comma } \\
\text { OR } \\
\text { (b) Okereke, DG Taskforce }\end{array}$ \\
\hline
\end{tabular}




\begin{tabular}{|c|c|c|c|c|}
\hline & & & & $\begin{array}{l}\text { on Prohibition of Importation } \\
\text { of Arms, speaks on Adamu, } \\
\text { new Police IG. (Note: double } \\
\text { commas used to mark off } \\
\text { common noun in apposition). }\end{array}$ \\
\hline 33 & $\begin{array}{l}* 2019 \text { election: AD } \\
\text { disowns, withdraws } \\
\text { support for Oyo guber } \\
\text { candidate, Hakeem } \\
\text { Alao (January 17, } \\
2019) \text {. }\end{array}$ & $\begin{array}{l}\text { Two persons referred to } \\
\text { here: Hakeem Alao and Oyo } \\
\text { guber } \\
\text { (Ambiguity). }\end{array}$ & $\begin{array}{l}\text { Only one person } \\
\text { called Hakeem Alao } \\
\text { who is the Oyo guber } \\
\text { candidate, is the one } \\
\text { disowned by AD } \\
\text { which also withdraws } \\
\text { support for him. }\end{array}$ & $\begin{array}{l}\text { (a) } 2019 \text { election: Ad } \\
\text { disowns, withdraws support } \\
\text { for Oyo guber candidate } \\
\text { Hakeen Alao. (Note: no } \\
\text { comma) } \\
\text { OR } \\
2019 \text { election: Ad disowns, } \\
\text { withdraws support for } \\
\text { Hakeem Alao, Oyo guber } \\
\text { candidate. (Note: a comma } \\
\text { separates Alao and Oyo } \\
\text { guber candidate, the } \\
\text { common noun in apposition). }\end{array}$ \\
\hline 34 & $\begin{array}{l}\text { *2019 election: New } \\
\text { IG of Police, } \\
\text { Mohammed Adamu } \\
\text { warns officers ahead of } \\
\text { massive deployment } \\
\text { (January 17, 2019) }\end{array}$ & $\begin{array}{l}\text { Two persons referred to } \\
\text { here: Mohammed Adamu } \\
\text { and New IG of Police. } \\
\text { (Ambiguity). }\end{array}$ & $\begin{array}{l}\text { Only one person } \\
\text { called } \\
\text { Adamu who is the } \\
\text { new IG of Police is } \\
\text { meant. }\end{array}$ & $\begin{array}{l}\text { (a) } 2019 \text { election: New IG of } \\
\text { Police Mohammed Adamu } \\
\text { warns officers ahead of } \\
\text { massive deployment. (Note: } \\
\text { no comma) } \\
\text { OR } \\
\text { (b) } 2019 \text { election: } \\
\text { Mohammed Adamu, new IG } \\
\text { of Police, warns officers } \\
\text { ahead of massive } \\
\text { deployment. (Note: double } \\
\text { commas). }\end{array}$ \\
\hline 35 & $\begin{array}{l}* 2019 \text { election: Don’t } \\
\text { allow Buhari to destroy } \\
\text { our democracy, he is not } \\
\text { trustworthy - Ezekwesili } \\
\text { begs Nigerians (January } \\
17,2019) \text {. }\end{array}$ & $\begin{array}{l}\text { Comma wrongly used to } \\
\text { separate two independent } \\
\text { clauses. (Comma splice). }\end{array}$ & & $\begin{array}{l}\text { Don't allow Buhari t } \\
\text { destroy our democracy; he i } \\
\text { not trustworthy - Ezekwesil } \\
\text { begs Nigerians. (Note: semi } \\
\text { colon used to separat } \\
\text { independent clauses). }\end{array}$ \\
\hline 36 & $\begin{array}{l}\text { *Jonathan's ex-aid, } \\
\text { Omokri reacts as } \\
\text { Atiku, Saraki arrive } \\
\text { US. (January } 18,2019 \text { ) }\end{array}$ & $\begin{array}{l}\text { Two persons referred to } \\
\text { here: Omokri and Jonathan's } \\
\text { ex-aid. (Ambiguity). }\end{array}$ & $\begin{array}{l}\text { Only one person } \\
\text { called Omokri who is } \\
\text { Jonathan's ex-aid is } \\
\text { meant. }\end{array}$ & $\begin{array}{l}\text { (a) Jonathan's ex-aid Omokr } \\
\text { reacts as Atiku, Saraki arrive } \\
\text { US. (Note: no comma after } \\
\text { ex-aid as a proper nour } \\
\text { cannot be in apposition to } \\
\text { common noun). } \\
\text { OR } \\
\text { (b) Omokri, Jonathan's ex } \\
\text { aid, reacts as Atiku, Sarak } \\
\text { arrive US. (Note: double } \\
\text { commas after Omokri). }\end{array}$ \\
\hline 37 & $\begin{array}{l}\text { *Nigeria } \\
\text { minister, } \\
\text { Kebbirion: }\end{array}$ & $\begin{array}{l}\text { Two persons referred to } \\
\text { here: Aliero and Ex-minister } \\
\text { (Ambiguity). }\end{array}$ & $\begin{array}{l}\text { Only one person } \\
\text { called Aliero, who is } \\
\text { an ex-minister, wins } \\
\text { Kebbi r central } \\
\text { Senatorial district. }\end{array}$ & $\begin{array}{l}\text { (a) Nigeria election: Ex- } \\
\text { minister Aliero wins Kebbi } \\
\text { Central Senatorial district. } \\
\text { (Note: no comma). } \\
\text { OR } \\
\text { (b) Aliero, ex-minister, wins } \\
\text { Kebbi Central Senatorial } \\
\text { district. (Note: double } \\
\text { commas marking of common } \\
\text { noun in apposition). }\end{array}$ \\
\hline 38 & $\begin{array}{l}\text { *Nigeria election: } \\
\text { Court of Appeal } \\
\text { President's husband, } \\
\text { Bulkachujwa is APC } \\
\text { senator elect for }\end{array}$ & $\begin{array}{l}\text { Two persons referred to } \\
\text { here: Bulkachuwa and Court } \\
\text { of Appeal } \quad \text { President's } \\
\text { husband. (Ambiguity). }\end{array}$ & $\begin{array}{l}\text { Only one person } \\
\text { called Bulkachuwa } \\
\text { who is the Court of } \\
\text { Appeal President's } \\
\text { husband is meant. }\end{array}$ & $\begin{array}{l}\text { (a) Nigeria election: Court of } \\
\text { Appeal President's husband } \\
\text { Bulkachuwa is APC senator- } \\
\text { elect for Bauchi North (Note: } \\
\text { no comma). }\end{array}$ \\
\hline
\end{tabular}




\begin{tabular}{|c|c|c|c|c|}
\hline & $\begin{array}{l}\text { Bauchi } \quad \text { North } \\
\text { (February 26, 2019). }\end{array}$ & & & $\begin{array}{l}\text { OR } \\
\text { (b) Bulkachuwa, Court of } \\
\text { Appeal President's husband, } \\
\text { is APC senator-elect for } \\
\text { Bauchi-North. (Note: double } \\
\text { commas marking common } \\
\text { noun in apposition.) }\end{array}$ \\
\hline 39 & $\begin{array}{l}\text { *Nigeria election: } \\
\text { PDP's Aduda floors } \\
\text { APC, emerges winner } \\
\text { for FCT senatorial } \\
\text { election (February 26, } \\
2019\end{array}$ & $\begin{array}{l}\text { Comma wrongly used to } \\
\text { separate independent clauses. } \\
\text { (comma splice) }\end{array}$ & & $\begin{array}{l}\text { Nigeria election: PDP's } \\
\text { Aduda floors APC; emerges } \\
\text { winner of FCT } \\
\text { Senatorial election. (Note: } \\
\text { semi-colon used to separate } \\
\text { independent clauses). }\end{array}$ \\
\hline 40 & $\begin{array}{l}\text { *2019 election results: } \\
\text { Ex-APC presidential } \\
\text { aspirant Ogbonnia tells } \\
\text { Atiku what to do. } \\
(\text { February } 27,2019)\end{array}$ & $\begin{array}{l}\text { Two persons referred to } \\
\text { here: Ogbonnia and Ex-APC } \\
\text { presidential aspirant. The } \\
\text { presence of the singular verb } \\
\text { tells saves the ambiguity. }\end{array}$ & 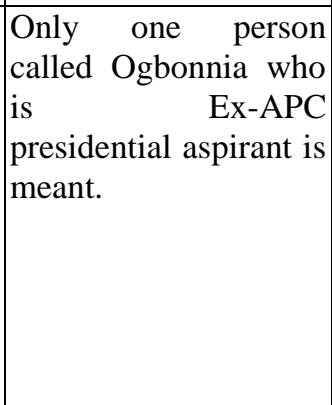 & $\begin{array}{l}\text { (a) } 2019 \text { election results: Ex- } \\
\text { APC presidential aspirant, } \\
\text { tells Atiku what to do. (Note: } \\
\text { no comma). } \\
\text { OR } \\
\text { (b) } 2019 \text { election results: } \\
\text { Ogbonnia, ex-APC } \\
\text { presidential aspirant, tella } \\
\text { Atiku what to do (Note: } \\
\text { double commas). }\end{array}$ \\
\hline 41 & $\begin{array}{l}\text { *Presidential elections: } \\
\text { How Buhari's victory } \\
\text { 'll affect Nigeria - } \\
\text { Jonathan's ex-aid, } \\
\text { Omokri. (February 28, } \\
\text { 2019). }\end{array}$ & $\begin{array}{l}\text { Two persons referred to } \\
\text { here: Omokri and Jonathan's } \\
\text { ex-aid. (Ambiguity). }\end{array}$ & $\begin{array}{l}\text { Only one person } \\
\text { called Omokri who is } \\
\text { Jonathan's ex-aid is } \\
\text { meant. }\end{array}$ & $\begin{array}{l}\text { (a) Presidential election: } \\
\text { How Buhari's victory 'll } \\
\text { affect Nigeria - Jonathan's } \\
\text { ex-aid Omokri. (Note: no } \\
\text { comma) } \\
\text { OR } \\
\text { (b) Presidential election: } \\
\text { How Buhari's victory 'll } \\
\text { affect Nigeria - Omokri, } \\
\text { Jonathan's ex-aid. (Note: a } \\
\text { comma after Omokri) }\end{array}$ \\
\hline 42 & $\begin{array}{l}\text { *Buhari vs Atiku: Von } \\
\text { DG, Okechukwu slams } \\
\text { PDP over rejection of } \\
\text { presidential results } \\
\text { (February 28, 2019). }\end{array}$ & $\begin{array}{l}\text { Two persons referred to } \\
\text { here: Okechukwu and Von } \\
\text { DG, but the presence of the } \\
\text { singular verb slams tends to } \\
\text { prevent the error of } \\
\text { Ambiguity. }\end{array}$ & $\begin{array}{l}\text { Only one person } \\
\text { called Okechukwu } \\
\text { who is the DG of Von } \\
\text { slams PDP. }\end{array}$ & $\begin{array}{l}\text { (a) Von DG Okechukwu } \\
\text { slams PDP over rejection of } \\
\text { presidential results. (Note: no } \\
\text { comma) } \\
\text { OR } \\
\text { (b) Okechukwu, Von DG, } \\
\text { slams PDP over rejection of } \\
\text { presidential results. (Note: } \\
\text { double commas marking off } \\
\text { common noun). }\end{array}$ \\
\hline 43 & $\begin{array}{l}\text { *Oyo decides: PDP } \\
\text { candidate, Makinde } \\
\text { wins } 27 \text { out of } 33 \text { LGS. } \\
\text { (March 10, 2019) }\end{array}$ & $\begin{array}{l}\text { Two persons referred to } \\
\text { here: Makinde and PDP } \\
\text { candidate, but the presence } \\
\text { of the singular verb wins } \\
\text { tends to prevent the error of } \\
\text { Ambiguity. }\end{array}$ & $\begin{array}{l}\text { Only one person } \\
\text { called Makinde who is } \\
\text { PDP candidate wins } \\
27 \text { out of } 33 \text { LGS. }\end{array}$ & $\begin{array}{l}\text { (a) Oyo decides: PDP } \\
\text { candidate Makinde wins } 27 \\
\text { out of } 33 \text { LGS. (Note: no } \\
\text { comma). } \\
\text { OR } \\
\text { (b) Oyo decides: Makinde, } \\
\text { PDP candidate, wins } 27 \text { out } \\
\text { of } 33 \text { LGS. (Note: double } \\
\text { commas mark off common } \\
\text { noun in apposition. }\end{array}$ \\
\hline 44 & 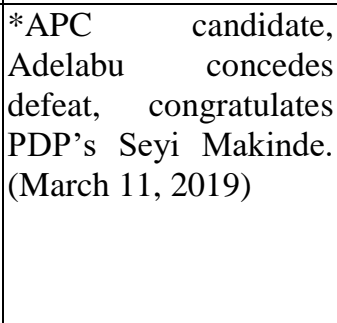 & $\begin{array}{l}\text { Two persons referred to } \\
\text { here: Adelabu and APC } \\
\text { candidate, but the presence } \\
\text { of the singular verbs } \\
\text { concedes and congratulates } \\
\text { tend to prevent the error of } \\
\text { Ambiguity. }\end{array}$ & $\begin{array}{l}\text { Only one person } \\
\text { called Adelabu who is } \\
\text { APC r candidate } \\
\text { concedes defeat and } \\
\text { congratulates PDP's } \\
\text { Seyi Makinde. }\end{array}$ & $\begin{array}{l}\text { (a) APC candidate Adelabu } \\
\text { concedes } \\
\text { congratulates PDP's Seyi } \\
\text { Makinde. (Note: no comma } \\
\text { after common noun } \\
\text { candidate). } \\
\text { OR } \\
\text { (b) Adelabu, APC candidate, }\end{array}$ \\
\hline
\end{tabular}




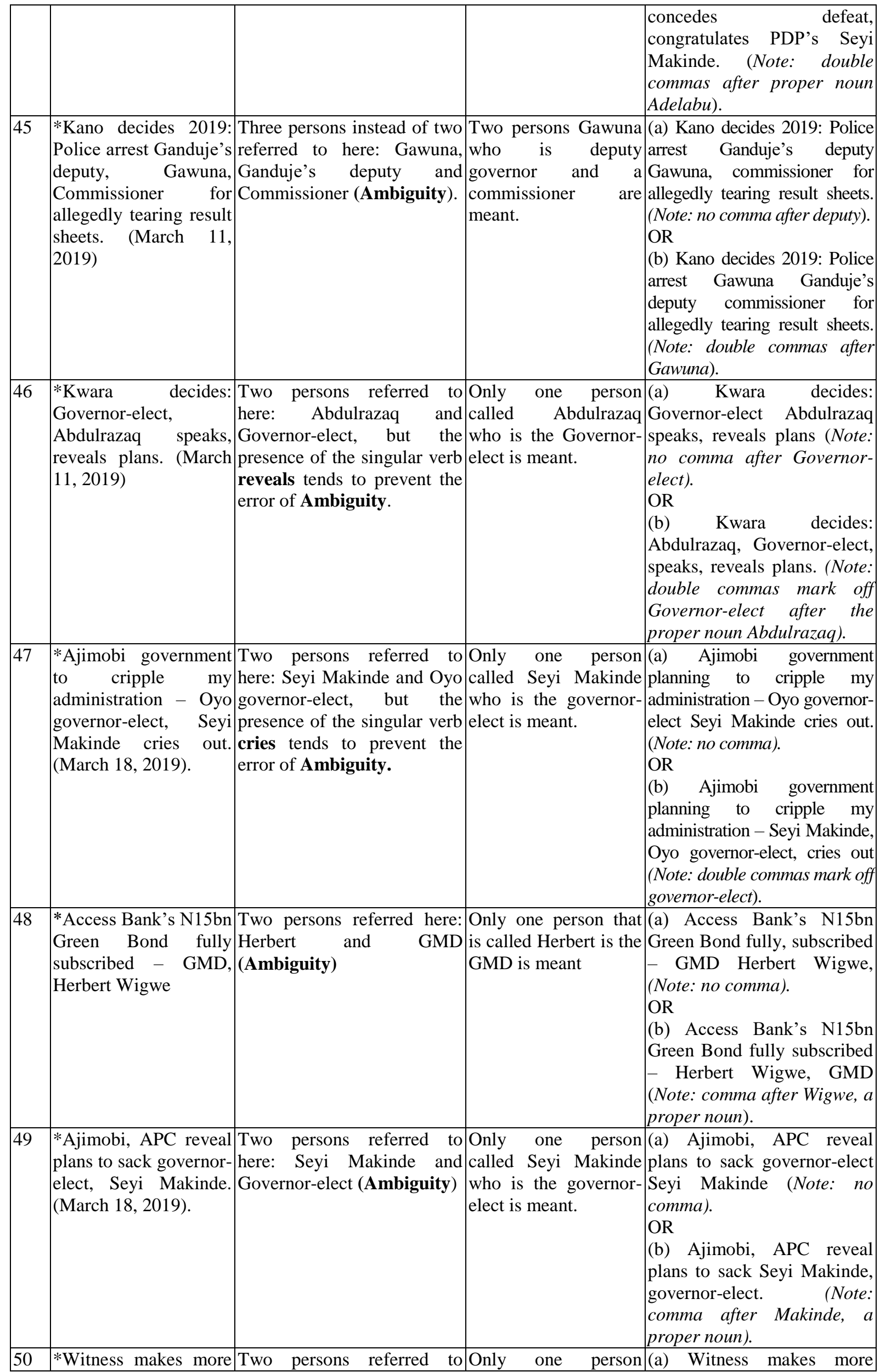


revelation on former here: Ehindero and former called Ehindero who revelation on former Police Police IG, Ehindero’s Police IG (Ambiguity). $\quad$ is former Police IG is IG Ehindero’s $¥ 300 \mathrm{~m}$ fraud $¥ 300 \mathrm{~m}$ fraud case (March 21, 2019). meant. $\quad$ case (Note: no comma). OR

(b) Witness makes more revelation on Ehindero, former Police IG's, $¥ 300 \mathrm{~m}$ fraud case. (Note: double commas mark off former Police IG, the common noun in apposition to the proper noun Ehindero).

\section{DISCUSSION}

Social deviance is not criminality. While a social deviant is not a criminal, it cannot be denied that his activities can bring shame and ridicule to himself and members of his family and community. This is the case with punctuation (never a calm subject, crystal, 2015) and writing, especially in this age of digital journalism.

While wrong punctuation might not attract any criminal penalty, it surely can attract the anger and odium of discerning readers whose taste it offends by making comprehension difficult through attraction of additional unintended meanings. Using punctuation poorly or wrongly is akin to wearing clothes inside out. Although the clothes would cover the nakedness they are intended to cover, the wearer certainly attracts funny and uncomplimentary comments from the audience because his action is against accepted societal norm or practice. Wrong punctuation is, thus, part of the linguistic infelicities that should be avoided in writing in both print and digital journalism.

In the purposively sampled fifty faulty headlines above, two major errors associated with the wrong use of the comma were identified as ambiguity and the comma splice. Ambiguity occurs when a sentence or headline is open to two or more interpretations instead of just one. In the preceding table, the headlines afflicted with errors of ambiguity are $1,2,5,6,7,8,9,10,14,15,16,17,18,19,21,24$, $26,27,28,29,30,32,33,34,36,37,38,41,45,48$ and 49.

In these headlines, the wrong use of the comma causes them to attract additional unintended meanings as explained in column II. Thus, like every mechanical tool or implement, punctuation marks as safety valves of written communication (Balogun, 1995) have their rules of usage. Their use or application is not haphazard. Just as a 10/11 spanner cannot be used to tighten or loosen a 12/13 bolt, so can a single comma not be used in place of double commas. Among the rules of application of the comma is: use double commas to mark off a noun in apposition (Strunk and White, 1974, 2009; Balogun, 1995; Yagoda, 2012; Crystal, 2015; Miller-Broomfield, 2016; Vietor, 2017). This rule of application is still extent and has not been repealed.

In English grammar, it is a common noun that is usually in apposition to a proper noun and not the other way round. This grammatical rule too is still extant and has not been abrogated in the age of digital journalism. In the headlines listed above, this grammatical rule was never observed; hence the error of ambiguity set in and caused the comma to go into a coma.

The comma splice error is found in headlines numbers 11, 12, 13, 20, 22, 23, 25, 31, 35 and 39. As the table shows, these headlines do not attract additional unintended meanings, but tend to offend against linguistic good manners. According to Blue (2000), there are times when a comma splice is a justifiable stylistic device and not an error. One of such times is if the independent clauses are very short and especially if the subject is the same for both clauses. This is the case with these headlines above.

Although they do not have a change in meaning different from the intended, which makes them justifiable stylistic devices (Blue, 2000), the argument of this paper is that in headlines such devices should be minimized or avoided completely as they tend to force extra burden on the comma thus sending it to a coma. Such stylistic devices could be reserved for the body of the story, especially in digital journalism. Second, it does appear that with this constant resort to justifiable stylistic device, the semi-colon is being forced into extinction. The semi-colon need not be rendered useless by the willingness of the comma to be over-burdened because as Miller-Broomfield (2016) observed, in the 
world of (informal) electronic communication, however, punctuation conventions are rapidly changing and evolving (or devolving), depending on one's perspective. Headlines do not fall under "informal electronic communication"; hence the argument against justifiable stylistic device" in headlines.

The reconstructed headlines (column IV) bring out neatly the meanings intended without the fear of attracting unintended additional meaning, no matter how the headlines are read. This is the result of correct or proper use of punctuation, especially the comma: communication breakdown is avoided! The paper, thus, agrees with the views of Yagoda (2012), Raphel (2015) and Oluwadoro (2017). Punctuation is the salt in the soup of writing.

\section{CONCLUSION AND RECOMMENDATIONS}

\subsection{Conclusion}

Communication, the main purpose of language, can always breakdown if the ingredients and components of language are not appropriately, proportionately and adequately deployed and harnessed. Punctuation, one of the ingredients of language, especially writing (written communication) is one ingredient that does not seem to be getting attention by researchers as the literature search revealed. It is always assumed (wrongly though) that everyone knows it, which led Balogun (1995), (2018) to observe that the comma is the most widely used, but the least wisely used.

This paper, using a sample of fifty faulty headlines taken from Daily Post (http://dailypost.us9.listmanage.comm) on-line newspaper, has shown that a lot can go wrong in writing if punctuation is not properly handled. Specifically, it focused on the comma, which wrong application or use can result in errors of ambiguity and the comma splice - obstacles to effective communication. Like any piece of mechanical device that requires bolts and nuts to hold it together or safety valves to prevent a collapse or an explosion, writing (written communication) depends on some ingredients for it to be effective. These are punctuation marks.

As the $21^{\text {st }}$ century has witnessed and is still witnessing a reconstruction of the English Language in order to serve the communication needs of the globalised world and to disseminate information via the information and communication technology (Tunde-Awe, 2014), it cannot be gainsaid that punctuation will always be relevant as the safety valves of written communication or the spanners needed to tighten and loosen the bolts and nuts in writing.

\section{RECOMMENDATIONS}

Reporters, editors, proof-readers and indeed all digital journalism practitioners should familiarize themselves with rules of punctuation even though they may have their various house styles for their various tabloids. Rules of punctuation are superior to various house styles the way a national constitution is superior to any other law in a nation.

All tabloids whether print, electronic or digital should, as a matter of priority, employ applied linguists who specialize in writing in their organizations or at least engage them as consultants as is the case with Prof Ben Yagoda of the University of Delaware and the New York Times.

\section{REFERENCES}

[1] Azuike, M.N. (2014). Language, Literature and Communication in the Globalized and Digital Age. In Adekoya, S., Taiwo, R., Ayoola, K., and Adegoju, A. (eds) Current Linguistic and Literary Issues in Digital Communication in the Globalized Age. Ile-Ife: Obafemi Awolowo University Press.

[2] Balogun, F.U. (1995). Punctuation marks as safety valves in Written Communication. Journal of Liberal Studies. Vol. 5, No. 2. Pp $151-157$

[3] Balogun, F.U. (2018). Social Media and the Teching/Leqrning of Writing in Secondary Schools in AkokoEdo Local Government Area, Edo State. Unpublished Research Project, National Teachers Institute (NTI) Kaduna, Nigeria.

[4] Blue, T. (2000). When is a comma splice Not an Error? Available at: https://grammartips.homestead.com. Accessed 25/9/19.

[5] Crystal, D. (1987). The Cambridge Encyclopedia of Language. Cambridge: Cambridge University Press.

[6] Crystal, D. (2015). Lousy at punctuation? Fear not - so was Wordsworth. The Guardian. Available at: https://www.theguardian.com. Accessed 14/9/19. 
[7] Duskova, A.L. (1969). On the Sources of Errors in Foreign Language Learning. International Review of Applied Linguistics. 7: $11-30$.

[8] Gleason, H.A. Jr (1968). Contrastive Analysis in Discourse Structure. Monograph Series on Languages and Linguistics 21. (Georgetown University, Institute of Languages and Linguistics) [Reprinted in Maleleani and Lockwood, 1973: 258 - 76]

[9] James, C. (1998). Errors in Language Learning and Use. London: Longman

[10] Lado, R. (1957). Linguistics Across Cultures. Ann Arbor: University of Michigan Press.

[11] Lado, R. (1964). Language Teaching: A Scientific Approach. McGraw Hill, New York.

[12] Miller-Broomfield, C. (2016). Punctuation in the Digital Age. Unravel. Available at: https:// unravellinguage.com. Accessed 25/9/19.

[13] Oluwadoro, J.O. (2016). Concord Error in Postgraduate Dissertation and Theses. International Journal of English Language and Literature Studies 5(1): 1 - 9.

[14] Oluwadoro, J.O. (2017). Error in Analysis of Long Essay and Theses' Acknowledgement by Nigerian Students. Akingba Journal of Linguistics and Literatures. No. 8, Issue 2. Pp 1 - 11).

[15] Raphel, A. (2015). A History of Punctuation for the Internet Age. The New Yorker. Available at: https://newyorker.com

[16] Stockwell, R. (1968). Contrastive Linguistics and Lapsed Time. In Alatis 111 - 26

[17] Starkey, L. (2004). How to Write Great Essays ( $1^{\text {st }}$ ed) New York: Learning Expren

[18] Strunk Jnr. W. and White, E.B. (1974, 2009) (5 $5^{\text {th }}$ ed). The Elements of Style. Boston: Allyson \& Bacon.

[19] Tunde-Awe, B.M. (2014). Nativization of English Language in a Multilingual Setting. The example of Nigeria. Academic Journal of Interdisciplinary Studies. Vol. 3, No. 6 pp. 85 - 92.

[20] Vie tor, A. (2007). A Short History of Punctuation (and why it's an essential part of communication). Available at: https://veracontent.com. Accessed 13/9/19.

[21] Wlikipedia. The Free Encyclopedia. https://en.n.wikipedia.org

[22] Yagoda, B. (2012). The Most Comma Mistakes. New York Times. Available at https://opinonator.blogs. nytimes.com/2012/06/31/the most.com. Accessed 3/19/2019, 3:31 PM

Citation: Femi U. Balogun. Comma in a Coma in Online Headlines: The Case of Daily Post. "International Journal on Studies in English Language and Literature (IJSELL), vol 7, no. 11, 2019, pp. 16-28 doi: http://dx.doi.org/10.20431/2347-3134.0711003.

Copyright: (C) 2019 Authors. This is an open-access article distributed under the terms of the Creative Commons Attribution License, which permits unrestricted use, distribution, and reproduction in any medium, provided the original author and source are credited. 